Nota Técnica

Pensar en Movimiento:

Revista de Ciencias del Ejercicio y la Salud

ISSN 1659-4436

Vol. 10, No.1, pp. $1-4$

\title{
NOTA TÉCNICA: EL ESTRÉS POR CALOR AMBIENTAL EN EL FÚTBOL AUMENTA EN SUPERFICIES SINTÉTICAS
}

\author{
Luis Fernando Aragón Vargas, Ph.D., FACSM \\ luis.aragon@ucr.ac.cr \\ Catedrático, Escuela de Educación Física y Deportes, Universidad de Costa Rica
}

Manuscrito recibido: 25/06/2011; aceptado: 23/02/2012; publicado: 30/06/2012

\begin{abstract}
RESUMEN
Aragón-Vargas, L.F. (2012). Nota técnica: El estrés por calor ambiental en el fútbol aumenta en superficies sintéticas. Pensar en Movimiento: Revista de Ciencias del Ejercicio y la Salud, 10 (1), 1-4. El estrés por calor ambiental ocurre por una combinación de la temperatura, la radiación y la humedad relativa del ambiente. Cuando se practica el fútbol en canchas sintéticas no techadas, la radiación solar produce un aumento importante de la temperatura de la superficie de juego. En esta nota técnica se explica cómo se calcula el estrés por calor según el índice WBGT (el cual no mide directamente la temperatura de la superficie de juego), y se documenta la magnitud del aumento en la temperatura de una superficie sintética, comparada con zacate natural en el mismo lugar. Se recomienda evitar la práctica del fútbol en las horas más calientes y con mayor radiación solar del día en este tipo de superficies.
\end{abstract}

Palabras claves: Termorregulación; calor ambiental; césped artificial

\section{ABSTRACT}

Aragón-Vargas, L.F. (2012). Technical paper: Environmental heat stress in football is increased in synthetic surfaces. Pensar en Movimiento: Revista de Ciencias del Ejercicio y la Salud, 10 (1), 14. Environmental heat stress is the result of ambient temperature, radiation, and relative humidity. During football (soccer) practice on synthetic surfaces and no roof, solar radiation causes an important temperature increase of the playing surface. This technical note explains how heat stress is calculated according to the WBGT index (which does not measure playing surface temperature directly), and quantifies the increase in a synthetic surface compared to natural grass on the same site. Football practice should be avoided during those hours with the highest solar radiation and temperature on this type of surface.

Key Words: Thermoregulation; heat stress; artificial turf; soccer 
Es bien conocido que cuando se practica deporte bajo condiciones de alto estrés por calor ambiental ocurre un impacto negativo sobre el rendimiento deportivo (Aragón Vargas, MoncadaJiménez, Hernández, Barrenechea, \& Monge, 2009; Maughan, Watson, \& Shirreffs, 2007); dicho impacto es mayor conforme aumenta la intensidad del ejercicio y la duración de este. Más aún, las consecuencias para la salud de los jugadores podrían ser negativas, al punto de convertirse en un problema de salud pública (Armstrong, 2003).

El índice más utilizado para medir el estrés por calor al que está sometida una persona y para definir los límites o rangos dentro de los cuales se puede realizar ejercicio sin exponerse a un alto riesgo, se conoce internacionalmente como WBGT (las siglas de su nombre en inglés: Wet Bulb Globe Temperature). La fórmula (1) para calcular el WBGT recomendada por el Colegio Americano de Medicina Deportiva en el año 2007 (ACSM por sus siglas en inglés) (Armstrong et al., 2007) es una modificación de la que publicaron Yaglou y Minard 50 años antes (Yaglou \& Minard, 1957). Esta fórmula es un promedio ponderado de tres temperaturas ambientales:

$$
\text { (1) } W B G T=\left(0.7 T_{b h}\right)+\left(0.2 T_{g}\right)+\left(0.1 T_{b s}\right)
$$

Donde:

- $\mathbf{T}_{\text {bh }}$ es la temperatura de bulbo húmedo natural expuesto al sol, sin ventilar,

- $\mathbf{T}_{\mathbf{g}}$ es la temperatura de globo negro con exposición directa al sol,

- $\mathbf{T}_{\mathrm{bs}}$ es la temperatura de bulbo seco, la cual debe obtenerse a la sombra.

Existe una alternativa (2) sumamente útil cuando no se cuenta con el dato de temperatura de globo negro, como en el caso de la mayoría de los reportes de servicios meteorológicos. Ante eso se debe utilizar la fórmula de Gagge y Nishi (1976):

Donde:

(2) $\mathrm{WBGT}=\left(0.567 \mathrm{~T}_{\mathrm{bs}}\right)+\left(0.288 \mathrm{P}_{\mathrm{a}}\right)+3.38$

- $\mathbf{T}_{\text {bs }}$ es la temperatura de bulbo seco reportada por el servicio meteorológico, y

- $\mathbf{P}_{\mathrm{a}}$ es la tensión de vapor en $\mathrm{mmHg}$ o Torr.

Como la tensión de vapor se reporta generalmente en hectoPascales (la unidad de presión del sistema internacional se conoce como Pascal), entonces es necesario hacer la conversión tomando en cuenta que 1 Torr $=133.3224 \mathrm{~Pa}=1.333224 \mathrm{hPa}$. Además, resulta preciso advertir que todavía algunos libros de texto y otras fuentes utilizan la fórmula equivocada de WBGT $=\left(0.567 \mathrm{~T}_{\mathrm{bs}}\right)+(0.393$ $\left.P_{a}\right)+3.94$, error generado en la década de 1980 y el cual exagera el verdadero índice de estrés por calor ambiental.

Una vez obtenido el valor de WBGT para un lugar y momento determinado, se puede comparar con las recomendaciones de distintas organizaciones, para tener una idea del nivel de riesgo de sufrir complicaciones por calor si se practica deporte en esas condiciones. Según la declaración de consenso del ACSM (Armstrong et al., 2007), si el valor de WBGT es superior a $28^{\circ} \mathrm{C}$ se considera que el riesgo de complicaciones por calor (incluidos el golpe de calor, y el agotamiento por calor) es muy alto. 
Es importante en este contexto indicar que en Costa Rica, debido a la programación frecuente de juegos alrededor del mediodía, no resulta extraño registrar valores de WBGT claramente superiores a ese: con la ayuda de un monitor de ambiente térmico QuesTemp $36^{\circledR}$ (Quest Technologies, Oconomowoc, WI, USA) se registraron las temperaturas durante el desarrollo de algunos juegos oficiales de fútbol de primera división. Así, se han obtenido los siguientes resultados (promedio \pm D.E.): mes de abril, estadio de Nicoya al mediodía, WBGT = $31.9 \pm 1.2^{\circ} \mathrm{C}$; diciembre, estadio de Liberia entre 3 y 5 p.m., WBGT $=26.8 \pm 1.0^{\circ} \mathrm{C}$; abril, estadio de Puntarenas al mediodía, WBGT $=31.9 \pm 1.4^{\circ} \mathrm{C}$; abril, estadio de Guápiles al mediodía, WBGT $=30.5 \pm 0.4^{\circ} \mathrm{C}$. Este índice objetivo de estrés térmico sugiere que los jugadores están compitiendo en condiciones adversas con un alto riesgo de complicaciones por calor.

Para complicar la situación, la temperatura de la superficie de juego es un factor que podría incidir en el estrés por calor de los jugadores de fútbol, el cual no está contemplado por el índice WBGT. Los jugadores profesionales se quejan de la alta temperatura de las superficies sintéticas donde practican el fútbol, particularmente cuando brilla el sol. Por lo tanto, cabría preguntarse: ¿es cierto que la superficie sintética es más caliente que el zacate o césped natural, o se trata de una percepción debido al mejor agarre de los zapatos y por ende mayor coeficiente de fricción en las canchas sintéticas?

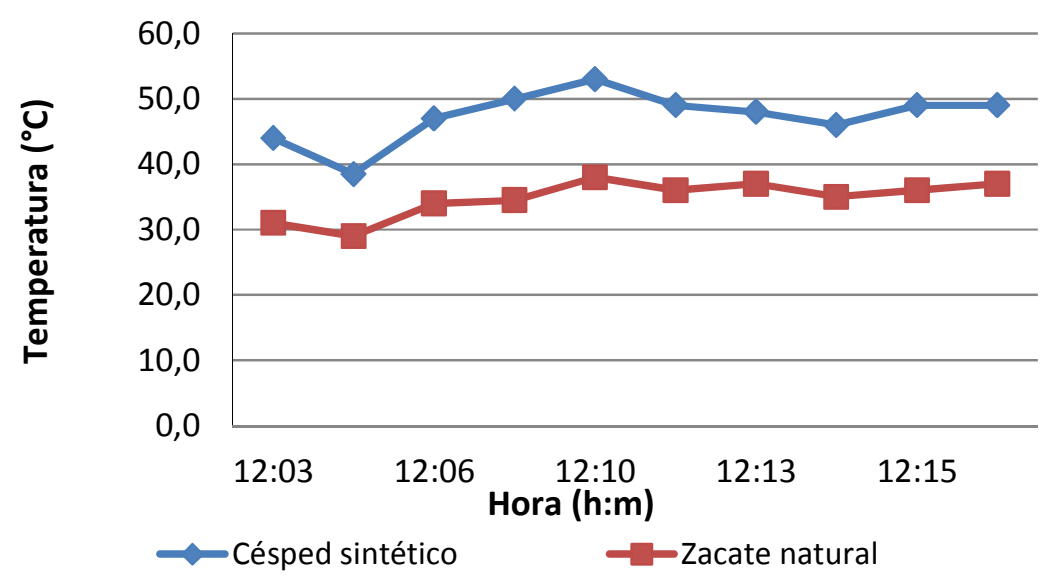

Figura 1. Comparación de temperatura de la superficie de juego. La medición se hizo en un día soleado (parcialmente nublado), el promedio del zacate natural fue $34.8^{\circ} \mathrm{C}$ y del césped sintético fue $47.4^{\circ}$; $p<0.0001$.

La Figura 1 muestra los resultados de diez mediciones realizadas en la cancha pedagógica de la Universidad de Costa Rica. Para ello se colocaron termómetros de bulbo seco debidamente calibrados sobre la superficie de juego, simultáneamente en zacate natural y en césped sintético, a diez metros de distancia entre sí y a mediodía con cielo ligeramente nublado. La temperatura fue 12.6 $\pm 1.8^{\circ} \mathrm{C}$ más alta en el césped sintético $(p<0.0001)$. 
Tabla 1

Temperaturas superficiales en la cancha de fútbol

\begin{tabular}{ccccc}
\hline & \multicolumn{2}{c}{ Zacate natural } & \multicolumn{2}{c}{ Césped sintético } \\
\cline { 2 - 5 } & Media & D.E. & Media & D.E. \\
\hline Soleado & $34.8^{\circ} \mathrm{C}$ & $2.8^{\circ} \mathrm{C}$ & $47.4^{\circ} \mathrm{C}$ & $3^{3.9} \mathrm{C}$ \\
Nublado & $20.0^{\circ} \mathrm{C}$ & $0.0^{\circ} \mathrm{C}$ & $20.3^{\circ} \mathrm{C}$ & $0.4^{\circ} \mathrm{C}$ \\
\hline
\end{tabular}

Al repetir la prueba en el mismo lugar, pero en un día completamente nublado, la diferencia no fue significativa $\left(0.3 \pm 0.4^{\circ} \mathrm{C}, \mathrm{p}=0.052\right)$. La tabl a 1 muestra los promedios y desviaciones estándar de la temperatura para cada día y cada superficie; la interacción entre día y superficie fue significativa $(F=537.8, p<0.0001)$.

Ante esto, se concluye que las canchas de fútbol con superficie sintética se calientan significativamente más que las de césped natural como respuesta a la radiación solar. Las altas temperaturas de las superficies sintéticas podrían agravar el estrés por calor ambiental que experimentan los jugadores de fútbol al practicar o competir en días soleados; se recomienda, por lo tanto, evitar las horas más calientes y con más radiación solar del día.

\section{Referencias}

Aragón Vargas, L.F., Moncada-Jiménez J., Hernández J., Barrenechea A., \& Monge M. (2009). Evaluation of pre-game hydration status, heat stress, and fluid balance during professional soccer competition in the heat. European Journal of Sport Science, 9(5), 269-276.

Armstrong, L.E., Casa, D.J., Millard-Stafford, M., Moran, D.S., Pyne, S.W., \& Roberts, W.O. (2007). American College of Sports Medicine position stand. Exertional heat illness during training and competition. Medicine \& Science in Sports \& Exercise, 39(3), 556-72.

Armstrong, L.E. (Editor). (2003). Exertional Heat Illnesses. Champaign, Illinois: Human Kinetics..

Gagge A. P. \& Nishi Y. (1976). Physical indices of the thermal environment. ASHRAE Journal, 18, 4751.

Maughan, R.J., Watson, P., \& Shirreffs, S.M. (2007). Heat and cold: what does the environment do to the marathon runner? Sports Medicine, 37(4-5), 396-399.

Yaglou C. P. \& Minard D. (1957). Control of Heat Casualties at Military Training Centers. American Medical Association Archives of Industrial Health, 16, 302-316.

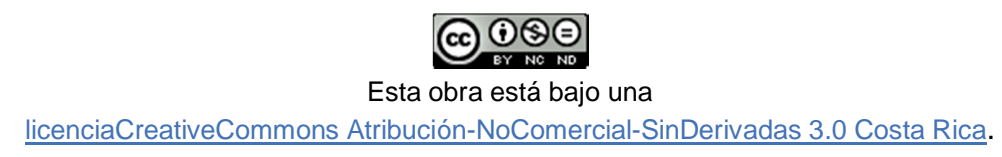

